\title{
KOMMENTAR
}

\section{Der Staat als Arbeitgeber}

\section{Karin Gottschall Markus Promberger}

Eine Anstellung im Staatsdienst - das wünschten sich Generationen zumindest für ihre Kinder. Beamte und öffentliche Arbeitnehmer werden zwar nicht reich, aber sie haben einen krisensicheren Arbeitsplatz. Auch für die Bürgerinnen und Bürger in Deutschland ist öffentliche Beschäftigung durch wohlfahrtsstaatliche Dienstleistungen in Gesundheit, Bildung, Erziehung und Arbeitsmarkt über Jahrzehnte zu einem festen Bestandteil von Existenzsicherung und Lebensqualität geworden. Und alle, einschließlich der Privatwirtschaft, profitieren bis heute von der umfassenden staatlichen Gewährleistung im Bereich der Infrastruktur.

Öffentliche Beschäftigung ist hierzulande bis in die 1990er Jahre ein wichtiger Stabilisierungsfaktor für Arbeitsmarkt und soziale Integration gewesen. Mit einem Anteil von weniger als $20 \%$ an der Gesamtbeschäftigung hatte die Bundesrepublik zwar im Vergleich zu Schweden, Frankreich und selbst Großbritannien immer schon einen eher kleinen öffentlichen Dienst. Gleichwohl erfüllte die Beschäftigungsstruktur über Jahrzehnte eine wichtige Vorbildfunktion für die Privatwirtschaft: durch die Angleichung von Arbeits- und Entgeltregelungen von Beamten und Tarifbeschäftigten, eine komprimierte Entgeltstruktur und die Integration von arbeitsmarktschwachen Gruppen.

Doch seither ist viel Wasser die Flüsse des rheinischen Kapitalismus hinabgeflossen, seine verteilungs-, interventions- und teilhabepolitischen Grundkonstellationen sind unter Druck geraten. Dabei steht der Staat auch als Arbeitgeber angesichts chronischer Finanzprobleme und veränderter Ansprüche der Bürger und Bürgerinnen vor besonderen Herausforderungen. Er soll Effizienz und Effektivität verbinden, Kostenersparnis und bessere Dienstleistungsqualität realisieren, Bürokratie abbauen und gleichzeitig konkurrenzfähigen professionellen Service bieten. Neue Leitbilder wie der schlanke, kosteneffektive Staat, Übergänge von hoheitlicher zu Dienstleistungsorientierung, Rationalisierung nach Taylor oder McKinsey und verschiedene Facetten von Privatisierung und Outsourcing sind nicht ohne Folgen für die Arbeit im öffentlichen Sektor geblieben.

Seit Mitte der 1990er Jahre ist die öffentliche Beschäftigung um ca. $20 \%$ geschrumpft, auch die Arbeitsverhältnisse selbst haben sich durch Abbau von sogenannten Privilegien und Erosion der tradierten Standards des Normalarbeitsverhältnisses verändert. Betroffen sind nicht nur Arbeitsamtmitarbeiter, Reinigungskräfte und Müllwerker, sondern auch der akademische Nachwuchs. Der Wandel der öffentlichen Arbeit ist umfassend; er betrifft Beschäftigungsumfang und Beschäftigungsformen, Lohnhöhen, Betriebsorganisation, Mitbestimmung und Arbeitsbedingungen und er stellt die tradierte Vorbildfunktion öffentlicher Beschäftigung infrage. So deutet sich in Untersuchungen zum Wandel der Entgeltstruktur eine Dualisierung zwischen Beamten und langjährigen Angestellten einerseits und Neueinsteigern andererseits an. Die Einführung von leistungsorientierten Entgeltbestandteilen, die vordergründig vor allem Motivation und Produktivität erhöhen sollen, ist ambivalent, zielt sie hintergründig doch auf Einsparungen, indem die Leistungsbestandteile nicht oder nur zum Teil auf die Altersbezüge angerechnet werden. Qualitätsstandards und Einheitlichkeit öffentlicher Beschäftigung erodieren auch durch den Ausbau bestimmter Formen geförderter Beschäftigung.

Was bedeutet dies für den Gewährleistungsauftrag des Staates und die gemeinwohlorientierte Produktion öffentlicher Güter? Auch wenn bisher kaum gesicherte Erkenntnisse über den $\mathrm{Zu}$ sammenhang von Beschäftigungsverhältnissen und Dienstleistungsqualität vorliegen, lassen die Destandardisierung und Differenzierung öffentlicher Beschäftigung im Hinblick auf Entgelt, Altersvorsorge und Arbeitsplatzsicherheit ebenso wie Dualisierungseffekte zwischen Insidern und Newcomern befürchten, dass die propagierten Reformziele nur bedingt erreicht werden und die Reformen selbst mit Nebenwirkungen und Risiken belastet sind.

Wo und in welcher Form wollen wir den Staat als Arbeitgeber? Der aktuelle Umbau des öffentlichen Dienstes gibt hier keine befriedigende Antwort. Der „Staatsdiener“ des „bürokratischen Versorgungsstaates" kann kaum mehr auf gesellschaftliche Unterstützung rechnen; doch auch das an der Privatwirtschaft orientierte Leitbild des „Dienstleisters“ im „schlanken Staat“ vermag nicht recht zu überzeugen. Denn der Staat handelt durch seine Beschäftigten. Er kann sich ebenso wie die Bürgerinnen und Bürger auf diese Handlungsressource nur verlassen, wenn öffentliche Beschäftigte kompetent, effizient und zuverlässig tätig sind. Privatisierung und Leistungsentlohnung können vielleicht Kosten sparen, aber ein privater Wachdienst ersetzt keine öffentliche Polizei. Wenn der Staat sich als der bessere Marktakteur etablieren will, wie etwa im Fall der privatwirtschaftlich agierenden Landesbanken, so verspielt er gesellschaftlichen Kredit und Steuergelder. Öffentliche Beschäftigung legitimiert und realisiert sich in der Herstellung öffentlicher Güter. Die Debatte, wie „staatsbedürftig“ die Gesellschaft und wie dem Gemeinwohl verpflichtete Beschäftigung zu gestalten ist, muss weiter geführt werden.

\footnotetext{
Prof. Dr. Karin Gottschall lehrt Soziologie an der Universität Bremen und ist Abteilungsleiterin am Zentrum für Sozialpolitik der Universität Bremen. e-mail: k.gottschall@zes.uni-bremen.de Dr. Markus Promberger leitet den Forschungsbereich "Erwerbslosigkeit und Teilhabe" am Institut für Arbeitsmarkt- und Berufsforschung (IAB) in Nürnberg. e-mail: markus.promberger@iab.de
} 